\title{
Research on the Cognizance Mechanism of Excellent Microfilm included in Scientific Research Achievement
}

\author{
$\mathrm{KAN} \mathrm{Di}^{1, \mathrm{a}}$ \\ ${ }^{1}$ School of Marxism, Shenyang Jianzhu University, Shenyang, Liaoning, China \\ a674589344@qq.com
}

\begin{abstract}
Microfilm has increasingly become an effective carrier of ideological and political education in universities as an important part of excellent network culture. It discusses the cognizance mechanism of excellent microfilm included in scientific research achievement from three aspects, including cognizance standard, cognizance method and cognizance grade of excellent microfilm, and it will provide reference and guidance for the true realization of excellent microfilm included in scientific research achievement.
\end{abstract}

Keywords: Microfilm, cognizance mechanism of scientific research achievement, cognizance standard, cognizance method, cognizance grade

\section{优秀微电影纳入科研成果的认定机制研究}

\author{
阚迪 ${ }^{1, a}$
}

1 沈阳建筑大学马克思主义学院，沈阳，辽宁，中国

a674589344@qq.com

\section{摘要}

阐释了微电影作为优秀网络文化的一个重要组成部分日益成为高校思想政治教育的一个有效载体, 从优秀微电 影的认定标准、认定方法和认定等级等三个方面探讨了优秀微电影纳入科研成果的认定机制, 为真正实现优秀 微电影纳入科研成果提供借鉴和参考。

关键词: 微电影, 科研成果认定机制, 认定标准, 认定方法

\section{1. 前言}

2015 年，中共中央办公厅、国务院办公厅印发 《关于进一步加强和改进新形势下高校宣传思想工 作的意见》。其中明确肯定了优秀网络文化在当前思 想政治教育中的积极作用。微电影作为优秀网络文化 的一个重要组成部分, 以其 “微时长、微成本、微周 期” [1] 的特点风生水起, 以其 “短小、精练、灵活” ${ }^{[2]}$ 的形式风靡于互联网, 以其有声有像的电影情节深 受学生的关注、喜爱甚至追捧, 并以星火燎原之势成 为高校思想政治教育的一个有效载体。因此, 基于思 想政治教育教师的科研成果统计和职务职称评聘体
系进行优秀微电影作品认定机制的研究也就提上了 日程。

优秀微电影纳入科研成果的认定机制包括优秀 微电影的认定标准、认定方法和认定等级等三个方 面。

\section{2. 优秀微电影的认定标准}

根据微电影的自身特征和思想政治教育的信息 传播规律, 从微电影的创意性、思想性、深刻性、现 实性和影响力等五个方面对优秀微电影进行评价。通 过设置评价体系的一级、二级指标，采用达标的方式 
对申报的微电影进行综合评价。

\section{1. 创意性}

微电影的创意性是指作品中所具有的创造性意 识或创新性意识, 它是一部微电影的灵魂。微电影是 “微型” 的电影, 是一种特殊的电影, 它首先要具有 一般优秀电影所具备的基本特征才能够成为一部优 秀的微电影。对于一部电影来说, 能否抓人眼球, 能 否具有较高的上座率, 创意是至关重要的。“微电影 的 “微” 决定了它必须在极短时间内迅速抓住观众的 眼球, 让观众有兴趣继续观看” ${ }^{[3]}$, 因此, 想要在有 限的时间内（几分钟到三五十分钟）运用电影的手法 感染观众、实现教育作用, 这必然对微电影的创意提 出了更高的要求。具体来说, 一部优秀微电影的创意 性表现为: 首先, 要具有新颖性, 就是在一部微电影 中体现了之前闻所未闻、见所未见的想法, 使观众眼 前一亮, 倍感新鲜; 其次, 要具有独特性, 就是这部 微电影不是抄袭的、也不是模仿的, 而是独一无二、 不可替代的; 最后, 要具有回味性, 就是一部微电影 在观看之后能够让观众印象深刻, 回味无穷, 念念不 忘, 产生持久的影响力和广泛的传播性。因此, 思想 政治教育工作者在微电影制作之初就要做好顶层设 计, 牢牢把好创意关、选题关, 注重微电影的新颖性、 独特性和回味性的紧密结合, 达到既能吸引观众又能 传播思想政治教育的双重目的。

采用定性评价的方法, 对微电影的创意性进行评 价。把微电影的创意性设为优秀、良好、一般 3 个等 级。根据申报微电影的新颖性、独特性和回味性来评 价微电影的创意性。

\section{2. 思想性}

微电影的思想性是指作品中所表现出的政治倾 向和思想意义, 它体现了一部微电影的政治立场。微 电影作品的传播 “由于发布者特定的价值观和政治倾 向而带有意识形态的烙印, 在传播过程中起着政治宣 传、价值批判、思想传播和行为影响等功能” ${ }^{[4]}$ 。因 此, 作为高校思想政治教育重要载体的微电影不仅是 一种艺术的传播方式, 更加承载了党和国家进行政治 方向引领、意识形态培育等重要使命。因此, 致力于 进行大学生思想政治教育的优秀微电影只有具备正 向的思想追求和崇高的价值取向, “促使其产生奋发 向上的激情和勇于向前的人生追求” ${ }^{[5]}$, 才是具有优 秀的特质。当前, 微电影的思想性具体表现为: 政治 立场鲜明、弘扬真善美、传播正能量、思想观点和价 值取向正确; 阐释理论热点难点、厘清错误思潮、坚 定学生对实现中国特色社会主义的道路自信、理论自 信、制度自信和文化自信; 体现立德树人基本导向。

采用定性评价的方法, 对微电影的思想性进行评 价。把微电影的思想性设为优秀、良好、一般 3 个等 级。根据申报微电影的政治立场、思想的深度和广度 来评价微电影的思想性。

\section{3. 深刻性}

微电影的深刻性是指作品中所体现出的深层次 的哲学思考, 它体现了一部微电影的内涵深度。微电 影的深刻性不是空洞的说教, 而是让观众通过形象思 维的方式认识世界和解释世界, 并在这一过程中启发 观众的认识和思考, 并学会运用马克思主义的世界观 和方法论来解决自身的实际困难和理论需求。如果微 电影只是停留在表面现象, 没有引发观众深层次的了 解和思考, 没有提高人们的理性思维, 那么它就缺乏 深刻性, 也丧失了其所承载的思想政治教育的功能和 使命。因此, 优秀微电影要有理论深度, 只有具备哲 理性、思辩性、启迪性的微电影，才具有优秀的特质。

采用定性评价的方法, 对微电影的深刻性进行评 价。把微电影的深刻性设为优秀、良好、一般 3 个等 级。根据申报微电影的哲理性、思辩性和启迪性的深 刻程度来评价微电影的深刻性。

\section{4. 现实性}

微电影的现实性是指作品中所体现出的对世界、 社会和人类自身现实问题的理性思考和深厚情感, 它 体现了一部微电影的应用价值。承载了思想政治教育 功能和使命的微电影必须要实现教化的功能, 而单纯 教化的微电影必然无法吸引观众的关注, 从而使微电 影失去生机和意义。因此, 微电影必须要满足观众的 现实需求, 这样观众才能够积极主动融入微电影, 微 电影也才能够潜移默化地教化观众。当前, 微电影的 现实性具体表现为: 首先, 要具有社会性, 就是微电 影要密切关注社会生活和现实人生并给予理性思考 和深层关怀, 歌颂光明、鞭挞丑恶、弘扬正气、彰显 正义, 担当起社会责任; 其次, 要具有时效性, 就是 微电影要紧密关注并迅速捕捉国家热点事件、社会焦 点问题和百姓热点话题, 从而引发观众对当下现实问 题的关注与思考; 最后, 要具有需求性, 就是微电影 要以观众的需求为出发点, 帮助学生解决在学习、生 活等现实生活中遇到的问题和困惑，从而增强观众的 代入感, 达到教育效果。因此, 只有具备社会性、时 效性和需求性的微电影才能直达观众内心, 产生情感 共鸣, 实现教育功能和价值。

采用定性评价的方法, 对微电影的现实性进行评 价。把微电影的现实性设为优秀、良好、一般 3 个等 级。根据申报微电影的社会性、时效性和需求性来评 价微电影的现实性。

\section{5. 影响力}

微电影的影响力指作品对观众的思想或行动所 起作用的程度。微电影影响力的大小取决于它的观众 观影规模和观众对微电影的反馈。观众观影规模是指 观看微电影的观众数量。“微电影好与差的评判标准 很大程度由点击率说了算” “ ${ }^{[6]}$, 微电影的点击量越高、 汶览次数越多, 表明其观众数量越大、传播范围越大, 
意味越多观众潜在地接受微电影的价值影响也就越 大, 微电影的影响力也越大。因此, 微电影的影响力 与其观众观影规模呈正向相关性。观众对微电影的反 馈是指观众观看微电影之后对微电影的反映。微电影 的传播属于群体传播模式, 其影响力在很大程度也取 决于观众的评论、收藏和转载。网络的交互性特征, 使得微电影可以在很短的时间内就可以获得观众的 直接评论, 评论次数越多, 表明该作品的吸引力越大, 知名度越高, 社会影响力也就越大。此外, 收藏和转 载次数也直接表明微电影的被认可程度和社会价值。 微电影被收藏和被转载的次数越多, 表明这一作品受 到越多关注, 传播范围也越广, 那么它的思想政治教 育影响力也就越大。因此, 评论、收藏和转载次数都 是衡量微电影影响力的重要量化指标。

目前, 微电影的汶览数量、评论数量、收藏和转 载数量都是可以通过网络技术直接获取的, 因此, 可 以通过考量点击量来考查观众的规模; 通过考量评 论、收藏和转载数量来考查观众的反馈。此外, 微电 影的转化应用情况, 也在较大程度上反映出微电影的 影响力。可把微电影的成果转化情况 (如获取奖励情 况、转载平台情况) 纳入认定体系中作为参考, 以此 综合评价微电影的影响力。

采用定量评价的方法对微电影的影响力进行评 价。分别按照微电影的点击数量、评论数量、收藏和 转载数量和成果转化情况进行优秀、良好、一般 3 个 等级的划分。考核工作组可根据具体情况进行数量范 围设置来评价申报微电影的影响力。优秀微电影认定 体系如表 1 所示。

表 1 优秀微电影认定体系

\begin{tabular}{|c|c|c|}
\hline $\begin{array}{l}\text { 一级 } \\
\text { 指标 }\end{array}$ & 二级指标 & 认定标准 \\
\hline \multirow{3}{*}{$\begin{array}{l}\text { 创意 } \\
\text { 性 A }\end{array}$} & 新颖性 A1 & $\begin{array}{c}\text { 创意新颖、抓人眼球、使人倍 } \\
\text { 感新鲜 }\end{array}$ \\
\hline & 独特性 A2 & $\begin{aligned} \text { 思路清晰、创意独一无二、不 } \\
\text { 可替代 }\end{aligned}$ \\
\hline & 回味性 A3 & $\begin{array}{c}\text { 故事生动丰富、让人印象深刻、 } \\
\text { 回味无穷 }\end{array}$ \\
\hline \multirow{2}{*}{$\begin{array}{l}\text { 思想 } \\
\text { 性 B }\end{array}$} & $\begin{array}{c}\text { 政治立场 } \\
\text { B1 }\end{array}$ & $\begin{array}{c}\text { 政治立场鲜明、弘扬真善美、 } \\
\text { 传播正能量、思想观点和价值 } \\
\text { 取向正确 }\end{array}$ \\
\hline & $\begin{array}{l}\text { 思想深度 } \\
\text { 和广度 B2 }\end{array}$ & $\begin{array}{c}\text { 阐释理论热点难点、厘清错误 } \\
\text { 思潮、充分发挥理论的先导作 } \\
\text { 用 }\end{array}$ \\
\hline \multirow{3}{*}{$\begin{array}{l}\text { 深刻 } \\
\text { 性 C }\end{array}$} & 哲理性 C1 & $\begin{array}{l}\text { 内涵丰富、哲理深刻、说理透 } \\
\text { 彻、引发人们深层次的思考 }\end{array}$ \\
\hline & 思辩性 C2 & $\begin{array}{l}\text { 提高人们的理性思维和对善 } \\
\text { 恶、真假、美丑的分辨能力 }\end{array}$ \\
\hline & 启迪性 C3 & $\begin{array}{c}\text { 启发人们的认识和思考, 帮助 } \\
\text { 人们解决困惑和问题 }\end{array}$ \\
\hline $\begin{array}{l}\text { 现实 } \\
\text { 性 D }\end{array}$ & 社会性 D1 & $\begin{array}{c}\text { 关注社会生活和现实人生并给 } \\
\text { 予理性思考和深层关怀 }\end{array}$ \\
\hline
\end{tabular}

\begin{tabular}{|c|c|c|}
\hline & 时效性 D2 & $\begin{array}{c}\text { 迅速捕捉国家热点事件、社会 } \\
\text { 焦点问题和百姓热点话题 }\end{array}$ \\
\hline & 需求性 D3 & $\begin{array}{c}\text { 以观众需求为出发点, 帮助解 } \\
\text { 决实际问题和困惑 }\end{array}$ \\
\hline \multirow{4}{*}{$\begin{array}{l}\text { 影响 } \\
\text { 力 } \mathrm{E}\end{array}$} & $\begin{array}{c}\text { 点击数量 } \\
\text { E1 }\end{array}$ & 达到 10000 次/年 \\
\hline & $\begin{array}{l}\text { 评论数量 } \\
\text { E2 }\end{array}$ & 达到 1000 次/年 \\
\hline & $\begin{array}{l}\text { 收藏和转 } \\
\text { 载数量 E3 }\end{array}$ & 达到 1000 次/年 \\
\hline & $\begin{array}{l}\text { 成果转化 } \\
\text { 情况 E4 }\end{array}$ & 获取奖励、转载平台情况 \\
\hline
\end{tabular}

\section{3. 优秀微电影的认定方法}

根据上述优秀微电影的认定标准, 着眼于客观、 公正、权威和操作便捷的原则, 采取网络学生评审和 专家最终评审相结合的方式进行优秀微电影的认定, 确定该网络作品是否满足优秀标准。

\section{1. 小组初审}

由审评小组对上交作品是否符合申报条件的基 本要求进行审查, 经审核合格的作品将统一上传至官 网进行展示。

\section{2. 学生初评}

（1）邀请校内不同学院、不同专业、不同年级 不少于 100 名学生组成初评小组, 对上传至官网的微 电影作品通过网站进行匿名投票评定。

（2）学生评委依据评定标准, 对申报的微电影 作品进行独立评价。依据微电影的具体情况, 给予“优 秀”、“良好”、“一般” 的等级评价。评定结果中, 有 $2 / 3$ 以上学生给予 “一般” 等级评价的作品将止 步于初评, 即无权进入专家终评。

\section{3. 专家终评}

（1）邀请不少于 5 名的高校宣传思想工作领域 中的专家, 组成专家小组, 对经过学生初评的微电影 进行最终认定。

（2）专家小组成员依据评定标准，对微电影进行 独立评价。对于达到二级指标要求标准的项目给予 “优秀” 等级。对于尚未达到优秀标准的二级指标项 目, 可依据微电影具体情况, 给予 “良好” 或 “一般” 的等级评价。每项二级指标只要有 $2 / 3$ 以上专家给 予 “优秀” , 该指标即可定为优秀, 其他情况可协商 确定。

\section{4. 优秀微电影的认定}

按照优秀微电影认定体系 15 个二级指标的认定 标准, 二级指标转化情况为 “优秀” 等级的申报作品, 
只要其二级指标为 “优秀” 等级的个数达到 10 个, 且无 “一般” 等级, 即可认定为 “优秀微电影” 。对 于二级指标转化情况为非 “优秀” 等级的申报作品, 其二级指标达到 “优秀” 等级的个数须为 12 个, 且 无 “一般” 等级, 方可认定为 “优秀微电影”。

\section{4. 优秀微电影的认定等级}

微电影作品获取奖励情况是认定网络作品优秀 性的一个客观评价指标, 可纳入科研成果的等级确认 体系。依据目前教育部思想政治工作司、国家互联网 信息办公室网络社会工作局开展的 “全国高校网络宣 传思想教育优秀作品评选” 活动或省级相关评选活动 的结果, 进行优秀微电影纳入科研成果的等级确认。 在 “全国高校网络宣传思想教育优秀作品评选” 活动 中被评为 “优秀微电影” 一等奖的作品, 可视为发表 在中文核心期刊上的文章, 认定为省 (部) 级科研成 果；在 “全国高校网络宣传思想教育优秀作品评选” 活动中被评为二、三等奖或在省级相关评选活动中被 评为一等奖的 “优秀微电影” , 可确认为省级行业科 研成果。

微电影作品转载平台情况也是认定网络作品优 秀性的一个客观评价指标, 可纳入科研成果的等级确 认体系。作品的转载平台按归属机构可以分为两种情 况: 一级媒体: 国家级权威主流电视、网络媒体; 教 育系统中有全国性影响力的电视、网络媒体; 国际主 流电视、网络媒体。被转载在一级媒体的微电影作品, 可视为发表在中文核心期刊上的文章, 认定为省 (部) 级科研成果。二级媒体: 省级主流电视、网络媒体 (包括中央级网络媒体地方频道)。被转载在二级媒 体的微电影作品, 可视为省级行业科研成果。

\section{5. 结论}

构建起优秀微电影纳入科研成果的认定标准、认 定方法和认定等级等认定机制, 是真正实现把优秀微 电影纳入科研成果的基础条件, 必将对优秀微电影的 不断发展提供重要保障, 也必将对思想政治教育教师 的科研成果统计和职务职称评聘体系起到极大的促 进作用。

\section{项目基金}

本文为中国建设教育协会教育教学科研 项目《高校校园景观文化对大学生思想政治教 育的作用及实现路径探究》 (2020068) 、辽宁 省教育厅科学研究项目 (1nqn201924) 阶段性 成果。

\section{REFERENCES}

[1] Liu, s.l.(2013) Analysis on the dissemination effect and related factors of Microfilm.Journal of Xihua University,1: 27-31.
[2] Li, 1.(2015) The Great Charm of microfilm--On theartistic creation of micro film.Knowledge of news, 11:86-87.

[3] Luo, h. m.(2013)Analysis on popularity and growth prospect of microfilm in "micro era".Southeast communication, 2:101-103.

[4] Kong, a.f.(2010) The Construction of University Network culture under the Field of Ideology.Journal of Changshu Institute of Technology, 12:13-14.

[5] Zhai, b.(2012)Research on the role of microfilm in promoting mainstream values. Journalism and Communication studies, 7:21-22.

[6] He, j. p, Zhang, w.(2013)Summary of the research status of "microfilm" in China.Contemporary film,6:129-133. 